\title{
Dengue haemorrhagic fever complicated with spontaneous psoas haematoma requiring blood products
}

${ }^{1}$ Sivakajani B, Ghetheeswaran S, Sanchayan T

Teaching hospital, Jaffna

\section{Abstract}

Dengue is a mosquito borne febrile viral disease .Sri Lanka is an endemic area where the incidence was peak in 2017 and slightly decreased in 2020 (28.7\%) because of preventive measures. Presentation can be asymptomatic or cause a spectrum of symptoms including undifferentiated viral fever, dengue fever, dengue haemorrhagic fever with or without shock and expanded dengue syndrome. We describe here a case of 48-years old man who presented to the emergency unit with three-day history of acute fever and prodromal features, subsequently diagnosed to have dengue haemorrhagic fever and it was complicated with psoas haemorrhage. Only limited cases were reported about spontaneous psoas muscle haemorrhage in dengue haemorrhagic fever in adult population.

\section{Introduction}

Dengue fever is a mosquito-transmitted disease and the virus belongs to the flavi virus family (1). It is an endemic to Sri Lanka (2) and can present with a wide range of clinical presentations ranging from a self-limiting simple dengue fever to lifethreatening dengue shock syndrome (1). In the recent years, atypical presentation of dengue cases was reported with more serious and fatal outcomes.

Patients with dengue illness can sometimes develop unusual manifestations such as involvement of liver, Kidney, brain and heart with or without evidence of fluid leakage; this is termed Expanded
Dengue Syndrome (EDS). The EDS is increasingly reported. Musculoskeletal system is also involved in Dengue fever. Spontaneous large muscle haematoma formation in Dengue Haemorrhagic fever (DHF) is rare. In most cases haematomas resolve spontaneously.

\section{Case presentation}

A 48-year-old male presented to the A \& E with generalized body ache and high fever (highest recorded temperature $39 \cdot 4^{\circ} \mathrm{C}$ ) of 3 days. On physical examination, he was found conscious (Glasgow Coma Scale 15), dehydrated with a heart rate 100/min, blood pressure 100/60 $\mathrm{mmHg}$. Dengue haemorrhagic fever (DHF) was diagnosed based on gall bladder wall oedema and free fluid in hepatorenal pouch and the patient was started on management as per the critical phase. At the mid-point of the critical phase he complained of a severe left sided groin and inguinal region pain. On examination the skin looked normal and there was no tenderness but he had severe pain when flexing the left thigh. All the pulses of the lower limb were present and neurological examination was normal. Significant initial laboratory investigations showed haemoglobin (Hb) $13.8 \mathrm{~g} / \mathrm{L}$ (13-15 g/L), haematocrit (Hct) $44 \%$, total white cell count (WBC) 4.4, platelet count (PC) 8, alanine aminotransferase (ALT) $48 \mathrm{U} / \mathrm{L}($ Normal $<40)$, aspartate aminotransferase (AST) 9 6U/L $($ Normal < 40), serum sodium $139 \mathrm{mmol} / \mathrm{L}(135-145)$, and C-reactive protein 
(CRP) $22.1 \mathrm{nmol} / \mathrm{L}(<6)$. PT 14 (11 to $13.5 \mathrm{~s}$ ), APTT 36 s (30-40). Blood picture: evidence of viral infection. Clinical suspicion of DHF was confirmed by positive anti-dengue-antibodies (IgM and $\mathrm{IgG}$ ). After the sudden development of the left thigh pain and an urgent USS showed a hypoechoic area involving the middle part of the left psoas. Appearances was compatible with bleeding into the left psoas with possible secondary infection (Figs.1\&2). Platelet on the day of development of haematoma was 4 , which was the lowest, recorded during his admission. The PT 14 (11 to $13.5 \mathrm{~s})$, APTT $36 \mathrm{~s}$ (30-40) and fibrinogen was 2 (1.5-4 g/L). The patient was transfused with $10 \mathrm{ml} /$ $\mathrm{kg}$ of red cells (2 pint blood), Fresh frozen plasma and Cryo precipitate due to reduction of PCV and tachycardia. The tachycardia settled and hematocrit picked up. He was also given IV ceftriaxone as there was evidence of secondary infection of the haematoma as the CRP rose to 75 . The patient improved gradually and was discharged home on the eleventh day of admission. He was followed-up after 1 month and was well and the repeat US scan showed no haematoma.

Fig 1- uss- haematoma in L/S psoas muscle

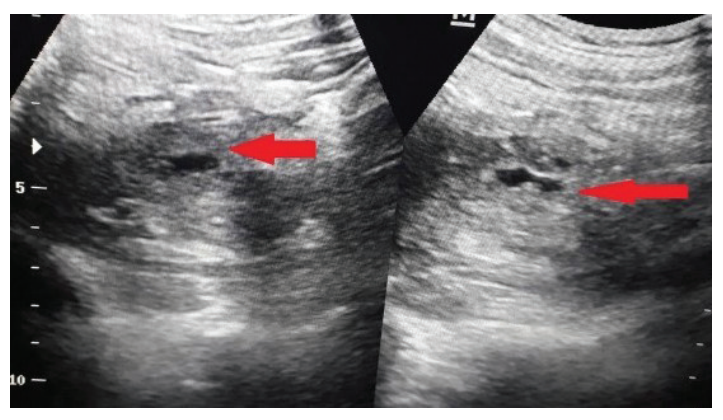

Fig 2- uss- haematoma in L/S psoas muscle

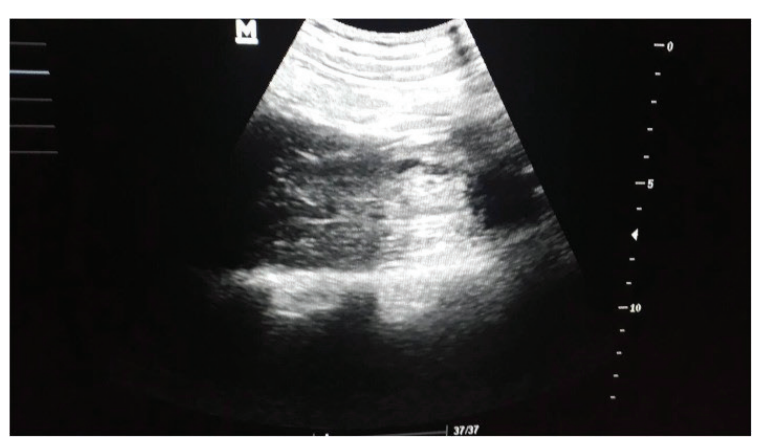

$-61-$

\section{Discussion}

The clinical features of Dengue fever and DHF are variable. DHF is becoming more common in Sri Lanka and physicians must be careful of its rare manifestations like muscle haematomas. These muscles haematomas can be in varied muscles: iliopsoas, psoas, retroperitoneal. To our knowledge rarely case of psoas haematoma has been reported. These consequences of haematoms can be varied. They could be asymptomatic, could lead to shock or may compress nerves leading to compression syndrome and retroperitoneal haematomas can be life threatening.

The exact pathogenesis of bleeding in dengue fever is still unknown. Thromboelastometry in patients with DHF have shown decrease of clotting factors and low platelet counts. The activation of the fibrinolytic mechanism may be a cause for spontaneous muscle haematomas in Dengue Haemorrhagic fever. There is some evidence of development of antibodies which might be cross reactive to plasminogen, which could be the reason for worsening of haemorrhage in DHF. We postulate that similar mechanisms would have led to the occurrence of haematoma in our patient as all coagulation tests were normal apart from platelet count.

The importance of recognizing muscle haemtomas is the prompt start of vigilant monitoring to prevent the patient form going into shock, by timely intervention of fluid resuscitation, blood transfusion if indicated and evacuation rarely if causing compression syndromes. Our patient did not have any neurological complications.

Most muscle haematomas in Dengue Haemorrhagic fever can be managed conservatively as most cases resolve spontaneously. Few cases have been managed with platelet transfusion since platelets have been very low. As in our case blood Jaffna Medical Journal 
transfusion was indicated as the patient was continuing to bleed in to the muscle resulting in PCV drop and hemodynamic instability.

\section{References}

1. Weekly Epidemiological Report, January 2018, Epidemiology unit SL. No title. Available from: http://www.epid.gov.lk/web/images/pdf/ wer/2018/vol_45_no_03-english.pdf.

2. Wijewickrama A, Fernando S, Bandara Jayerathne GS, Asela P, Abeynaike S, Gomes $\mathrm{L}$, et al. Emergence of a dengue virus serotype 2 causing the largest ever 2 dengue epidemic in
Sri Lanka short title: dengue serotype 2 causing a large outbreak in Sri Lanka running title: dengue serotype 2 causing massive outbreak in Sri Lanka. 10.1101/329318.

3. WHO . Comprehensive Guidelines for Prevention and Control of Dengue and Dengue Haemorrhagic Fever. 2011.

4. Sellahewa KH. Haematological disturbances in dengue Haemorrhagic fever - its pathogenesis and management perspectives.

5. Khan S, Gupta N, Maheshwari S. Acute gingival bleeding as a complication of dengue hemorrhagic fever. 